\title{
Palladium nanoparticles on carbon nanotubes as catalysts of cross- coupling reactions
}

\author{
Benedetta Cornelio, ${ }^{a, b}$ Graham A. Rance, ${ }^{* c}$ Marie Laronze-Cochard, ${ }^{b}$ Antonella Fontana, ${ }^{a}$ Janos Sapi, ${ }^{b}$ \\ Andrei N. Khlobystov*c
}

${ }_{5}$ Received (in $\left.X X X, X X X\right) X$ th $X X X X X X X X X 20 X X$, Accepted $X$ th $X X X X X X X X X 20 X X$
DOI: 10.1039/b000000x

The macroscopic properties of composite nanotube-nanoparticle superstructures are determined by a complex interplay of structural parameters at the nanoscale. The catalytic performance of different carbon nanotube-palladium nanoparticle catalysts, where nanoparticles were formed either directly onto

10 nanotubes or preformed prior to deposition on nanotubes using different types of surfactants, were tested in cross-coupling reactions. The decoration of multi-walled carbon nanotubes with preformed thiolatestabilised palladium nanoparticles yielded the optimum catalyst, exhibiting high activity and stability towards carbon-carbon bond formation and excellent recyclability, retaining high activity from cycle to cycle. The type of carbon nanotube support has pronounced effects on the density of deposited

15 nanoparticles, with more polarisable MWNT able to uptake the highest number of nanoparticles per unit surface area as compared to other carbon nanostructures (MWNT $>$ DWNT $>S W N T \sim$ GNF). Microscopic investigation of the nanoscale morphology found that nanoparticles increase in size during catalysis. The extent of growth is dependent on the type of nanocarbon support, with wider MWNT possessing lower curvature and thus retarding the growth and coalescence of nanoparticles to a greater extent than other 20 carbon nanostructures (SWNT $>>$ DWNT $>$ MWNT $\sim \mathrm{GNF}$ ). The type of halogen $\mathrm{X}$ in the C-X bond activated by palladium appears to influence the evolution of nanoparticles during catalysis, with $\mathrm{X}=\mathrm{Br}$ having the greatest effect as compared to $\mathrm{X}=\mathrm{Cl}$ or I. Overall, preformed thiolate-stabilised palladium nanoparticles deposited on MWNT from solution was found to possess the most functional catalytic properties, with optimum activity, stability and recyclability in a range of cross-coupling reactions.

\section{${ }_{25}$ Introduction}

The recovery and reuse of precious metal catalysts represents a key issue for the sustainable development of any catalytic process. Small metallic nanoparticles possess an extremely high surface-tovolume ratio and low coordination number of atoms at their

30 surfaces and are known to exhibit high catalytic performance in a variety of preparative chemical transformations. ${ }^{1}$ However, as nanoparticles are intrinsically metastable and tend to either irreversibly aggregate or leach atoms of metal under the conditions of standard chemical processes, leading to loss of intrinsic catalytic 35 performance, their practical application in catalysis is limited. ${ }^{2,3}$ One solution for stabilisation of metal nanoparticles involves the use of appropriate support materials. ${ }^{4-6}$ Although a number of different support materials have been investigated, including alumina, ${ }^{7}$ silica, ${ }^{8}$ metal oxides ${ }^{9}$ and layered double hydroxides, ${ }^{10}$ 40 the use of carbon nanostructures is attracting increasing attention as these high aspect ratio cylinders of $s p^{2}$-hydridised carbon possess attractive mechanical, chemical and thermal properties superior to other support materials, and impart both nanoparticle stability and a high degree of contact between the reactants of a 45 chemical process and the heterogeneous catalyst. ${ }^{11,12}$ Moreover, the combination of carbon nanostructures and different nanoscopic species within a composite material is expected to result in enhancement of their functional properties and can lead to new synergistic effects. ${ }^{13,14}$

50 The fabrication of superstructures of metallic nanoparticles and carbon nanotubes has been extensively explored and recently reviewed. ${ }^{15}$ Of the numerous experimental approaches investigated, the non-covalent deposition of metallic nanoparticles on carbon nanostructures is generally favoured as it permits ${ }_{55}$ preservation of the unique physicochemical properties of the underlying carbon nanostructure. ${ }^{6}$ Adsorption procedures can be categorised into ex situ and in situ approaches. ${ }^{16}$ The ex situ strategy involves the separate synthesis and deposition of preformed nanoparticles onto carbon nanostructures and is highly 60 attractive due to the vast number of routes for synthesis of metallic nanoparticles, affords nanoparticles of controlled size, shape, dispersity, composition and surface functionality and exploits a straightforward method for assembly of the hybrid structure with effective control of catalyst positioning and loading. ${ }^{17,18}$ In

65 contrast, in situ approaches involve simultaneous nucleation and growth of metal nanoparticles directly onto the carbon nanostructure sidewall. ${ }^{19}$ A number of different routes for 


\section{Cite this: DOI: $10.1039 / \mathrm{c0xx00000x}$}

metal ion reduction, including electrochemical methods, ${ }^{20,21}$ the use of supercritical carbon dioxide, ${ }^{22,23}$ microwave ${ }^{24}$ and thermal decomposition $^{25}$ and sputtering techniques, ${ }^{26}$ have been investigated in this approach, however nanoparticles generated by 5 these methods are typically polydisperse in terms of their physical characteristics, such as size and shape, and loading and distribution along the nanotube axis is often harder to control.

However, as carbon nanotubes and related cylindrical nanostructures are inherently structurally (distribution of lengths, 10 diameters, number of walls, defects) and chemically (functional groups, polarisability) inhomogeneous, methods for the preparation of nanotube-nanoparticle composites suffer from a lack of reproducibility. Hence, there is a real need to compare and contrast different approaches using a standardised set of carbon 15 nanotubes and evaluate their applicability as catalysts of a single preparative chemical reaction. Moreover, there is still a lack of fundamental knowledge regarding the effect of synergistic nanoparticle-nanotube interactions on catalytic activity and, therefore, the full scope of these nanomaterials in catalysis has yet 20 to be fully realised.

In this study, we compare ex situ and in situ methodologies for the preparation of composites of palladium nanoparticles and carbon nanotubes and assess the activity, selectivity and recyclability of the catalytic palladium centres in the Suzuki-

${ }_{25}$ Miyaura cross-coupling reaction. We demonstrate that our novel methodology for superstructure fabrication leads to nanocatalysts with remarkable performance in a range of chemical transformations and can be universally applied to different carbon supports with excellent retention of catalytic properties. 30 Furthermore, using a combination of advanced microscopy and spectroscopy techniques, we have correlated catalytic performance of composite materials with their nanoscale structures and uncovered key principles determining the importance of reagent selection to ensure the high efficiency and stability of the catalyst.

\section{${ }_{35}$ Experimental}

All reagents were purchased from Sigma-Aldrich, UK and used without further purification. Carbon nanostructures were purchased from a range of commercial suppliers and treated prior to use to minimise the content of residual metal catalyst from 40 synthesis $(\mathrm{S} 1 \dagger)$. All glassware was cleaned with a mixture of hydrochloric and nitric acid (3:1 v/v, 'aqua regia') and rinsed thoroughly with deionised water, cleaned with potassium hydroxide in isopropyl alcohol and finally rinsed thoroughly with deionised water. ${ }^{1} \mathrm{H}$ and ${ }^{13} \mathrm{C}$ NMR spectra were obtained using a 45 Bruker DPX-300 (300.13 and $75.48 \mathrm{MHz}$ respectively) spectrometer at $298 \mathrm{~K}$ using $\mathrm{CDCl}_{3}$ as the solvent. Mass spectrometry was conducted on either a Bruker Apex IV (ESI) or a Waters Autospec (EI) in positive mode. Thermogravimetric analysis was performed using a TA Instruments SDT Q600 under 50 a flow of air at a rate of $90 \mathrm{~mL} \mathrm{~min}-1$ at a heating rate of $10{ }^{\circ} \mathrm{C} \mathrm{min}-$
1 from room temperature to $1000{ }^{\circ} \mathrm{C}$. Transmission electron microscopy (TEM) was performed using a JEOL 2100F TEM (field emission gun source, information limit $<0.19 \mathrm{~nm}$ ) at room temperature. Analysis of nanoparticle size and density of adsorbed 55 nanoparticles on nanotubes was conducted using Gatan DigitalMicrograph software. Energy dispersive X-ray analysis was performed using an Oxford Instruments INCA 560 X-ray microanalysis system. TEM samples were prepared via dropdrying methanolic solutions onto a copper grid mounted "lacey" 60 carbon films. The palladium nanoparticle - carbon nanostructure catalysts were prepared according to modified literature procedures and novel protocols $(\mathrm{S} 1-\mathrm{S} 2 \dagger){ }^{27-33}$ Suzuki-Miyaura, Sonogashira and Heck cross-coupling reactions were performed according to modified literature protocols $(\mathrm{S} 3 \dagger) .{ }^{34-36}$

\section{${ }_{65}$ Results and discussion}

The synthesis of supported nanoparticle catalysts using bottom-up approaches permits discrete control of their macroscopic properties. Furthermore, the precise selection of suitable supports and stabilisers readily allows fine tuning of the features of the 70 resultant functional nanomaterial. For this study it was, therefore, essential to use a common carbon-based support material to negate the influence of the support itself. Multi-walled carbon nanotubes (MWNT) are an ideal support for metallic nanocatalysts as they possess high intrinsic polarisability suitable for nanoparticle 75 abstraction from bulk solution. Furthermore, their improved dispersion in the majority of solvents relative to narrower carbon nanotubes guarantees good access to the catalyst for the reactants of chemical reactions. Moreover, the cost of MWNT is relatively low and, as metals are not essential for their synthesis, the issue of 80 contamination from residual metal is largely averted. Therefore, in our study, we selected MWNT provided by Bayer Material Science (mean outer diameter of $13.7 \pm 3.8 \mathrm{~nm}$, lengths in the range 1-10 $\mu \mathrm{m}, 3-15$ layers of carbon) which possess a low number of functional groups $(\mathrm{S} 1 \dagger)$ as the model carbon support to assess the 85 applicability of different approaches for construction of palladium nanoparticle-carbon nanotube superstructures (Table 1).

Table 1 The MWNT/PdNP catalysts investigated in our study, the approaches adopted for their preparation and the nature of stabilising ligand around PdNP.

\begin{tabular}{|c|c|c|c|}
\hline Entry & Catalyst & Approach & Nanoparticle stabiliser \\
\hline 1 & MWNT/PdNP-1 & ex situ ${ }^{27}$ & dodecanethiol \\
\hline 2 & MWNT/PdNP-2 & ex situ ${ }^{28}$ & oleylamine \\
\hline 3 & MWNT/PdNP-3 & ex situ ${ }^{29}$ & 4-dimethylaminopyridine \\
\hline 4 & MWNT/PdNP-4 & in situ ${ }^{31}$ & none \\
\hline 5 & MWNT/PdNP-5 & in situ ${ }^{32}$ & none \\
\hline 6 & MWNT/PdNP-6 & in situ ${ }^{33}$ & sodium $n$-dodecylsulfate \\
\hline
\end{tabular}

90 The novel nanostructures MWNT/PdNP-1, MWNT/PdNP-2 and MWNT/PdNP-3 (entries 1-3, Table 1) were prepared by the 


\section{Cite this: DOI: $10.1039 / \mathrm{c0xx00000x}$}
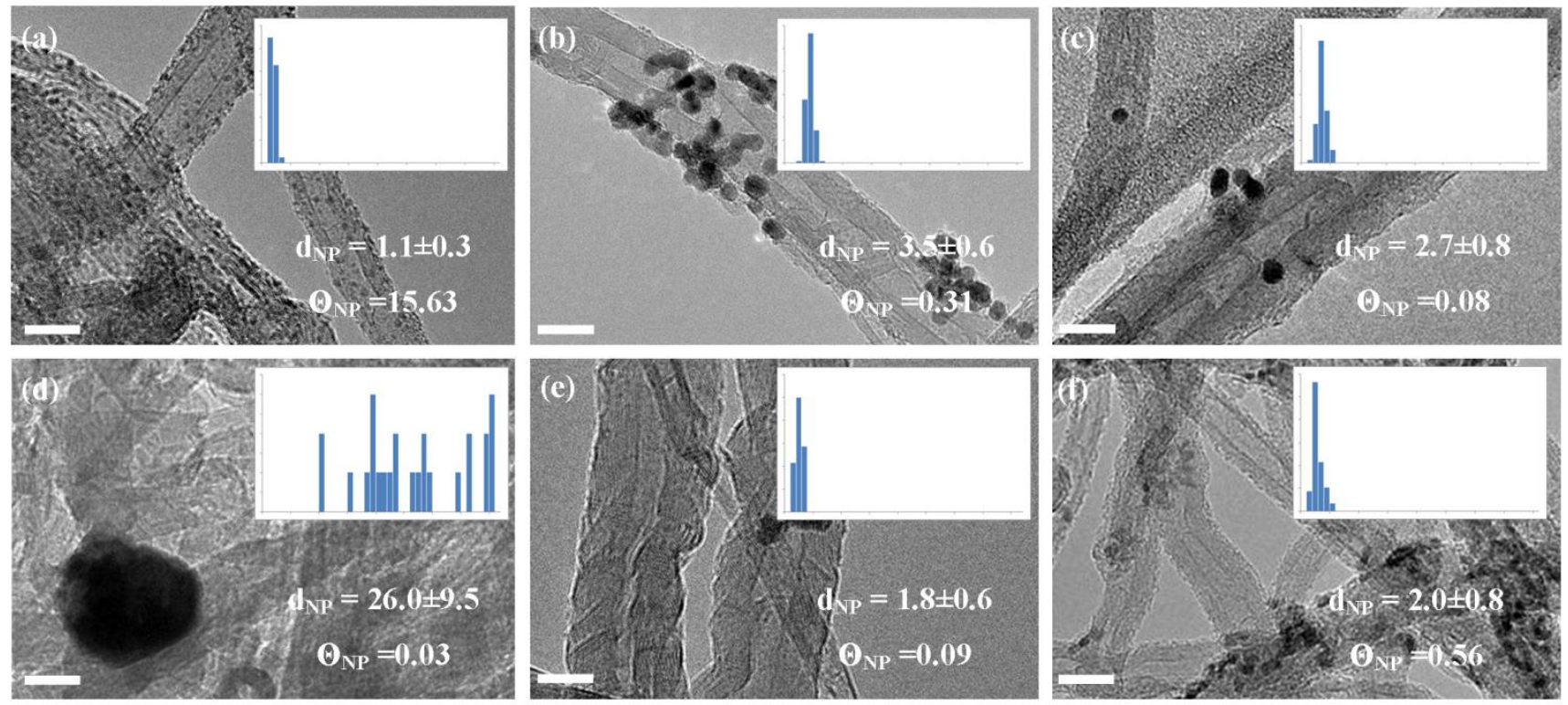

Fig. 1 Transmission electron microscopy (TEM) analysis of the MWNT/PdNP catalysts: (a) to (f) MWNT/PdNP-1 to MWNT/PdNP-6 respectively. $\mathrm{d}_{\mathrm{NP}}$ (in $\mathrm{nm})$ and $\Theta_{\mathrm{NP}}\left(\right.$ in $\left.10^{-2} \mathrm{~nm}^{-2}\right)$ refer to the mean nanoparticle diameter and surface density of nanoparticles on nanotubes respectively. Inset are the related histograms describing the distribution of nanoparticle diameters (range $=0-40 \mathrm{~nm}$ ). Scale bars are $10 \mathrm{~nm}$.

5 Table 2 Transmission electron microscopy (TEM) and thermogravimetric analysis (TGA) of the MWNT/PdNP catalysts: $\mathrm{d}_{\mathrm{NP}}$ and $\Theta_{\mathrm{NP}}$ refer to the mean PdNP diameter and surface density of PdNP on MWNT respectively as determined by TEM; $\mathrm{T}_{\text {oxid }}$ and $\mathrm{Pd}_{900}$ refer to the temperature of primary oxidation and the percentage of residual palladium metal at $800{ }^{\circ} \mathrm{C}$ respectively as determined by TGA.

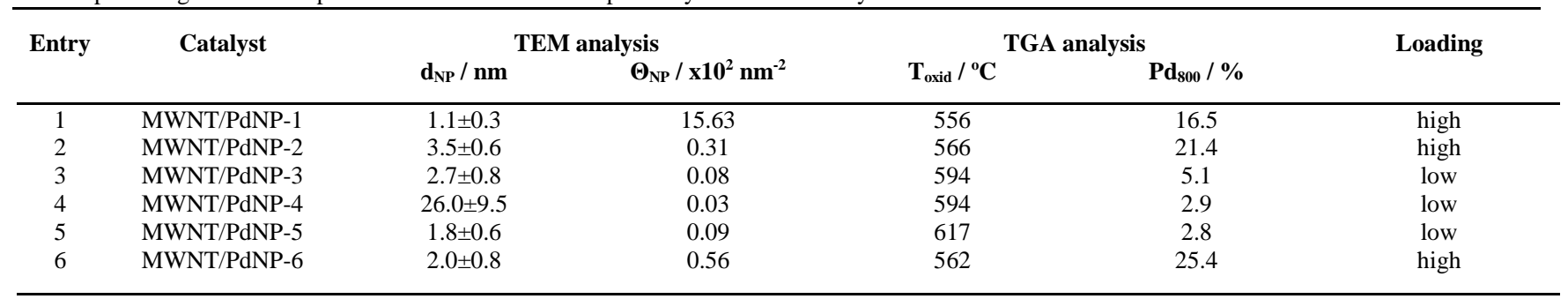

ex situ saturation of MWNT with preformed palladium 10 nanoparticles stabilised by dodecanethiol, ${ }^{27}$ oleylamine ${ }^{28}$ and 4 dimethylaminopyridine ${ }^{29}$ respectively, under conditions of ultrasonication. ${ }^{30}$ Conversely, MWNT/PdNP-4, MWNT/PdNP-5 and MWNT/PdNP-6 were obtained using modifications to reported literature in situ approaches: MWNT/PdNP-4 (entry 4,

${ }_{15}$ Table 1) in the absence of both stabiliser and reducing agent; ${ }^{31}$ MWNT/PdNP-5 (entry 5, Table 1) using solely a reducing agent ${ }^{32}$ and MWNT/PdNP-6 (entry 6, Table 1) in the presence of sodium $n$-dodecylsulfate as both the reducing agent and the stabiliser. ${ }^{33}$ All hybrid nanostructures were then characterised by a range of 20 analytical techniques (Fig. 1, Table 2 and S1-S2 $\dagger$ ).

TEM analysis of MWNT/PdNP-1 showed that very small spherical palladium nanoparticles $(1.1 \pm 0.3 \mathrm{~nm})$ are homogeneously distributed along the nanotube sidewall in high surface density $\left(15.63 \times 10^{-2} \mathrm{~nm}^{-2}\right)$. This was supported by a high
${ }_{25}$ percentage of elemental palladium $(16.5 \%)$ detected subsequent to MWNT oxidation by TGA. Conversely, the slightly larger nanoparticles in MWNT/PdNP-2 tended to cluster, presumably in suspension, which resulted in reduced mobility during the assembly procedure and, consequently, in a significant reduction 30 in the microscopically observed surface density on nanotubes and thermogravimetrically determined metal loading. As both of the preformed nanoparticles in these systems are prevented from aggregating by aliphatic chains, the observed difference in adsorption behaviour on nanotubes must be related to the bonding 35 between the head group of the stabiliser and the nanoparticle surface and suggests that the use of sulphur is more beneficial than nitrogen for assembly of MWNT/NP. This premise is further evidenced in MWNT/PdNP-3 where the electrostatic stabilisation of nanoparticle results in similar clustering of nanoparticles as in 


\title{
Cite this: DOI: $10.1039 / \mathrm{c0xx00000x}$
}

\author{
www.rsc.org/xxxxxx
}

ARTICLE TYPE

Table 3 Comparison of the selectivity, activity and recyclability of MWNT/PdNP, PdNP@Salk and the molecular catalyst $\mathrm{PdCl}_{2}\left(\mathrm{PPh}_{3}\right)_{2}$ in the SuzukiMiyaura cross-coupling of 1-halo-4-nitrobenzene $\mathbf{1}$ and phenylboronic acid $\mathbf{2}$ forming 4-nitro-1,1'-biphenyl $\mathbf{3}$ and nitrobenzene $\mathbf{4}$ as a dehalogenation byproduct, where $\mathrm{X}$ refers to the halogen substituent in the 1-position of the 1-halo-4-nitrobenzene (1a-c where $\mathrm{X}=\mathrm{Cl}, \mathrm{Br}$ and $\mathrm{I}$ respectively). ${ }^{a}$

\begin{tabular}{|c|c|c|c|c|c|c|c|}
\hline Entry & Catalyst & Cycle & $t / \mathrm{hr}$ & $\mathbf{X}$ & Conversion $/ \%^{b}$ & TOF $/ \mathrm{mol} \mathrm{mol}^{-1} \mathrm{hr}^{-1 c}$ & Selectivity $3: 4 / \%^{b}$ \\
\hline $1^{d, e}$ & $\mathrm{PdCl}_{2}\left(\mathrm{PPh}_{3}\right)_{2}$ & 1 & 18 & I & 94 & 1.0 & $85: 0$ \\
\hline $2^{f}$ & PdNP@Salk & 1 & 4 & I & 100 & 12.5 & $87: 13$ \\
\hline 3 & MWNT/PdNP-1 & 1 & 20 & $\mathrm{Cl}$ & 65 & 1.6 & $0: 100$ \\
\hline 4 & & 1 & 24 & $\mathrm{Br}$ & 100 & 2.1 & $100: 0$ \\
\hline 5 & & 2 & 24 & & 100 & 2.1 & $88: 12$ \\
\hline 6 & & 3 & 24 & & 100 & 2.1 & $100: 0$ \\
\hline 7 & & 4 & 48 & & 100 & 1.0 & $86: 14$ \\
\hline 8 & & 5 & 48 & & 32 & 0.3 & $100: 0$ \\
\hline 9 & & 1 & 4 & I & 100 & 12.5 & $80: 20$ \\
\hline 10 & & 2 & 4 & & 90 & 11.3 & $70: 30$ \\
\hline 11 & & 3 & 4 & & 100 & 12.5 & $71: 29$ \\
\hline 12 & & 4 & 10 & & 92 & 4.6 & $100: 0$ \\
\hline 13 & & 5 & 10 & & 100 & 5.0 & $80: 20$ \\
\hline $14^{g}$ & MWNT/PdNP-1* & 1 & 24 & I & 92 & 51.1 & $68: 32$ \\
\hline 15 & MWNT/PdNP-2 & 1 & 5 & I & 94 & 9.4 & $65: 35$ \\
\hline 16 & & 2 & 20 & & 94 & 2.4 & $57: 43$ \\
\hline 17 & MWNT/PdNP-3 & 1 & 20 & I & 78 & 2.0 & $100: 0$ \\
\hline 18 & & 2 & 5 & & 25 & 2.5 & $0: 100$ \\
\hline 19 & MWNT/PdNP-4 & 1 & 22 & I & 98 & 2.2 & $82: 18$ \\
\hline 20 & & 2 & 45 & & 83 & 0.9 & $71: 29$ \\
\hline 21 & MWNT/PdNP-5 & 1 & 9 & I & 94 & 5.2 & $86: 14$ \\
\hline 22 & & 2 & 6 & & 82 & 6.8 & $82: 18$ \\
\hline 23 & MWNT/PdNP-6 & 1 & 18 & I & 91 & 2.5 & $79: 21$ \\
\hline 24 & & 2 & 18 & & 0 & 0 & $0: 0$ \\
\hline
\end{tabular}

${ }^{a}$ Standard conditions: 1-halo-4-nitrobenzene 1 ( $0.04-0.50 \mathrm{mmol}, 1.0$ eq. $)$, phenylboronic acid 2 ( $0.05-0.6$ mmol, 1.2 eq. $)$, sodium acetate $(0.09-1.20$ $5 \mathrm{mmol}, 2.4$ eq.), palladium catalyst $(2 \mathrm{~mol} \%)$, methanol $(10-15 \mathrm{~mL}), 70{ }^{\circ} \mathrm{C} ;{ }^{b}$ determined by ${ }^{1} \mathrm{H}$ NMR spectroscopy; ${ }^{c}$ based on total metal loading; ${ }^{d} 5 \mathrm{~mol}$ $\%$ catalyst loading; ${ }^{e} 15 \% 1,1^{\prime}$-biphenyl detected; ${ }^{f}$ see $\mathrm{S} 1 \uparrow$ for details; ${ }^{g} 0.075 \mathrm{~mol} \%$ catalyst loading.

MWNT/PdNP-2 and a corresponding reduction in the amount of nanoparticles adsorbed to the nanotube sidewall. The total absence of a stabiliser in MWNT/PdNP-4 and MWNT/PdNP-5 yields both 10 highly size and shape polydisperse palladium nanoparticles and low loading, thus indicating that the associated in situ literature methods ${ }^{31,32}$ are not directly transferable to different carbon nanostructures. However, the use of sodium $n$-dodecylsulfate in the in situ preparation of MWNT/PdNP-6 afforded small spherical 15 nanoparticles consistent with those reported by Karousis et al., ${ }^{33}$ but significant nanoparticle aggregation and low nanoparticle density was observed. Hence, the ex situ approaches appear to permit more effective control of the physical features of nanoparticles and their corresponding interactions with MWNT, 20 with MWNT/PdNP-1 exhibiting structural characteristics most suitable for catalysis (e.g. small size and high loading) ${ }^{37}$

The Suzuki-Miyaura cross-coupling reaction is a very efficient and widely used method for the formation of carbon-carbon bonds. ${ }^{38-40}$ It is applicable to a wide range of substrates ${ }^{41}$ and has 25 become one of the most influential methods for the synthesis of natural products and pharmaceuticals. ${ }^{42-45}$ We have consequently chosen the Suzuki-Miyaura coupling as the model reaction to evaluate the catalytic performance of the hybrid MWNT-PdNP composites, using the coupling of 1-halo-4-nitrobenzene and ${ }_{30}$ phenylboronic acid as the standard reaction (Scheme $\mathbf{1}$ and Table 3).

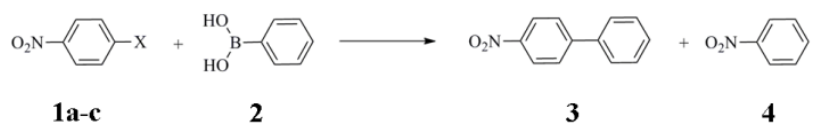

Scheme 1 The Suzuki-Miyaura and dehalogenation reactions (1a-c where $\mathrm{X}=\mathrm{Cl}, \mathrm{Br}$ and I respectively).

35 The reaction catalysed by $\mathrm{PdCl}_{2}\left(\mathrm{PPh}_{3}\right)_{2}$ and PdNP@Salk proceeds efficiently with excellent conversion and selectivity (entry 1-2, Table 3) and permitted identification of the afforded products by ${ }^{1} \mathrm{H}$ NMR spectroscopy. However, neither of these molecular or nanoparticulate catalysts can be recovered from the 40 reaction mixture by simple filtration and thus are not recyclable. MWNT/PdNP-1 gives excellent conversion of $\mathbf{1 c}$ and $\mathbf{2}$ and good selectivity for $\mathbf{3}$ and can be reused multiple times, retaining excellent catalytic performance (entries 9-13, Table 3). Conversely, MWNT/PdNP-2 exhibits good activity in the reaction 45 of $\mathbf{1 c}$ and $\mathbf{2}$ but moderate selectivity for $\mathbf{3}$ (entries 15-16, Table 3 ). Both excellent conversion and selectivity were seen using MWNT/PdNP-3 (entries 17-18, Table 3) in the initial cycle of catalysis, however, this decreased drastically after the first reuse. The catalytic performance of the in situ catalysts MWNT/PdNP-4, 50 MWNT/PdNP-5 and MWNT/PdNP-6 was also studied (entries 1924, Table 3) and showed good conversion and 


\section{Cite this: DOI: $10.1039 / \mathrm{c0xx00000x}$}
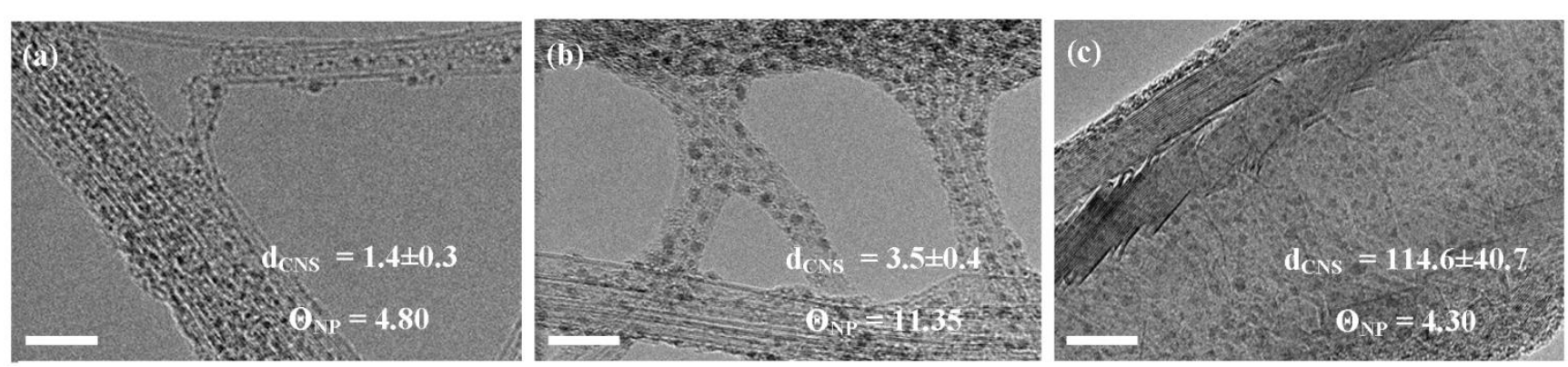

Fig. 2 TEM analysis of CNS/PdNP catalysts: (a) SWNT/PdNP-1; (b) DWNT/PdNP-1 and (c) GNF/PdNP-1. $\mathrm{d}_{\mathrm{CNS}}$ (in $\mathrm{nm}_{\text {) }}$ and $\Theta_{\mathrm{NP}}\left(\right.$ in $10^{-2} \mathrm{~nm}^{-2}$ ) refer to the mean nanostructure outer diameter and mean nanoparticle loading respectively. Scale bars are $20 \mathrm{~nm}$.

5 Table 4 TEM and TGA analysis of the CNS/PdNP catalysts.

\begin{tabular}{|c|c|c|c|c|c|c|}
\hline \multirow[t]{2}{*}{ Entry } & \multirow[t]{2}{*}{ Catalyst } & \multicolumn{2}{|c|}{ TEM analysis } & \multicolumn{2}{|c|}{ TGA analysis } & \multirow[t]{2}{*}{ Loading } \\
\hline & & $\mathbf{d}_{\mathrm{NP}} / \mathbf{n m}$ & $\Theta_{\mathrm{NP}} / \mathbf{x 1 0}^{2} \mathrm{~nm}^{-2}$ & $\mathbf{T}_{\text {oxid }} /{ }^{\circ} \mathbf{C}$ & $\mathbf{P d}_{800} / \%$ & \\
\hline 1 & SWNT/PdNP-1 & $1.1 \pm 0.3$ & 4.80 & 521 & 5.3 & low \\
\hline 3 & MWNT/PdNP-1 & & 15.63 & 556 & 16.5 & high \\
\hline 4 & GNF/PdNP-1 & & 4.30 & 695 & 4.1 & low \\
\hline
\end{tabular}

selectivity, but total loss of activity for MWNT/PdNP-6 was observed over the second catalytic run. Therefore, as MWNT/PdNP-1 appeared to be the most promising catalytic 10 system, we probed its properties further and discovered that it was remarkably effective for the coupling of the less reactive bromoderivative $\mathbf{1 b}$ (entries 4-8, Table $\mathbf{3}$ ), but unable to activate the corresponding chloro-derivative 1a (entry 3, Table $\mathbf{3}$ ), consistent with the known halogen reactivity scale for cross-coupling 15 reactions of $\mathrm{I}>\mathrm{Br}>\mathrm{Cl}$ and the reactivity-selectivity principle. Indeed, cross coupling with the most reactive iodo-derivative is less selective than with the bromo analogue. Furthermore, MWNT/PdNP-1 displayed excellent performance $(\mathrm{TOF}=51.1$ mol mol ${ }^{-1} \mathrm{hr}^{-1}$ ) under conditions of stress (entry 14, Table 3 ) and 20 is highly versatile, finding exceptional applicability towards the Suzuki-Miyaura reactions of electron-rich aryliodides (Table S2 in $\mathrm{S} 3 \dagger)$ and in the Sonogashira and Heck cross-coupling reactions $(\mathrm{S} 3 \dagger)$. MWNT/PdNP-1, therefore, represents the optimum catalyst for the formation of carbon-carbon bonds.

25 To probe the role of the underlying support, we next deposited thiolate-stabilised palladium nanoparticles onto alternate carbon nanostructures (CNS), specifically single- and double-walled carbon nanotubes (SWNT and DWNT respectively), which possess a concentric tubular structure analogous to that of MWNT, 30 and more topologically complex graphitised carbon nanofibers (GNF) which are a hybrid material comprising a series of stacked cups encased within concentric tubes (Fig. 2 and Table 4).

TEM revealed that our assembly method was universally applicable as the small palladium nanoparticles were shown to be
35 homogeneously distributed along all carbon nanostructures investigated. However, the relative affinity of the different carbon nanostructures for nanoparticles, characterised by both the PdNP surface density (determined by TEM) and total metal loading (determined by TGA), was found to be significantly different and 40 follows the pattern GNF SWNT $<$ DWNT $<$ MWNT. We have previously shown that the uptake of nanoparticles from suspension increases for larger and, therefore, more polarisable nanostructures and, consequently, the increased uptake correlating with the increase of the external diameter from SWNT to DWNT to MWNT 45 is consistent with enhanced dispersive nanoparticle-nanotube interactions. ${ }^{30}$ The adsorption capacity of GNF appears to be out of place in this trend as GNF have the largest diameter. However, considering that much of GNF consists of discontinuous cupstacked layers of carbon, which have lower polarisability than 50 continuous cylindrical layers of nanotubes, the polarisability of GNF and thus their affinity for nanoparticles should be expected to be substantially reduced as compared to DWNT and MWNT. Interestingly, excellent catalytic performance was retained for all carbon supports (Fig. 3 and S3 $\uparrow$ ), however, the MWNT/PdNP-1 55 catalysts required the shortest reaction times for full conversion of starting materials and therefore represents the most attractive catalytic material.

The fundamental catalytic performance of composite nanotubenanoparticle superstructures are determined by structural changes 60 at the nanoscale. The number, coordination and orientation of Pd surface atoms are of critical importance for the retention of catalytic activity measured at the macroscale and so we looked to 
compare the morphology of catalysts before and after the final catalytic cycle of the coupling reaction (Scheme 1) by TEM 5

(Table 5 and $\mathrm{S} 4 \dagger$ ).

Table 5 TEM analysis of CNS/PdNP catalysts before and after the final cycle of catalysis.

\begin{tabular}{|c|c|c|c|c|c|c|}
\hline \multirow[t]{2}{*}{ Entry } & \multirow[t]{2}{*}{ Catalyst } & \multirow[t]{2}{*}{$\mathbf{X}$} & \multicolumn{2}{|c|}{ Before catalysis } & \multicolumn{2}{|c|}{ After catalysis } \\
\hline & & & $\mathbf{d}_{\mathrm{NP}} / \mathbf{n m}$ & $\Theta_{\mathrm{NP}} / \mathbf{x 1 0}^{2} \mathrm{~nm}^{-2}$ & $\mathbf{d}_{\mathrm{NP}} / \mathbf{n m}$ & $\Theta_{\mathrm{NP}} / \mathrm{x10}^{2} \mathrm{~nm}^{-2}$ \\
\hline 1 & SWNT/PdNP-1 & I & $1.1 \pm 0.3$ & 4.80 & $5.7 \pm 1.9(\mathrm{x} 5.2 \uparrow)$ & $0.71(\mathrm{x} 6.8 \downarrow)$ \\
\hline 3 & MWNT/PdNP-1 & $\mathrm{Cl}$ & & 15.63 & $1.3 \pm 0.4(\mathrm{x} 1.2 \uparrow)$ & $9.69(\mathrm{x} 1.6 \downarrow)$ \\
\hline 4 & & $\mathrm{Br}$ & & & $2.0 \pm 1.9(\times 1.8 \uparrow)$ & $1.14(\mathrm{x} 13.7 \downarrow)$ \\
\hline 5 & & I & & & $1.3 \pm 0.9(\mathrm{x} 1.2 \uparrow)$ & $3.40(x 4.6 \downarrow)$ \\
\hline 6 & GNF/PdNP-1 & I & & 4.30 & $1.3 \pm 2.2(\mathrm{x} 1.2 \uparrow)$ & $1.00(\mathrm{x} 4.3 \downarrow)$ \\
\hline $9^{a}$ & MWNT/PdNP-4 & I & $26.0 \pm 9.5$ & 0.03 & - & - \\
\hline $10^{a}$ & MWNT/PdNP-5 & I & $1.8 \pm 0.6$ & 0.09 & - & - \\
\hline 11 & MWNT/PdNP-6 & I & $2.0 \pm 0.8$ & 0.56 & $4.0 \pm 1.1(\mathrm{x} 2.0 \uparrow)$ & $0.22(\mathrm{x} 2.8 \downarrow)$ \\
\hline
\end{tabular}

${ }^{a}$ No palladium detected post-catalysis. Fold changes in the nanoparticle size and distribution subsequent to catalysis are in parentheses.
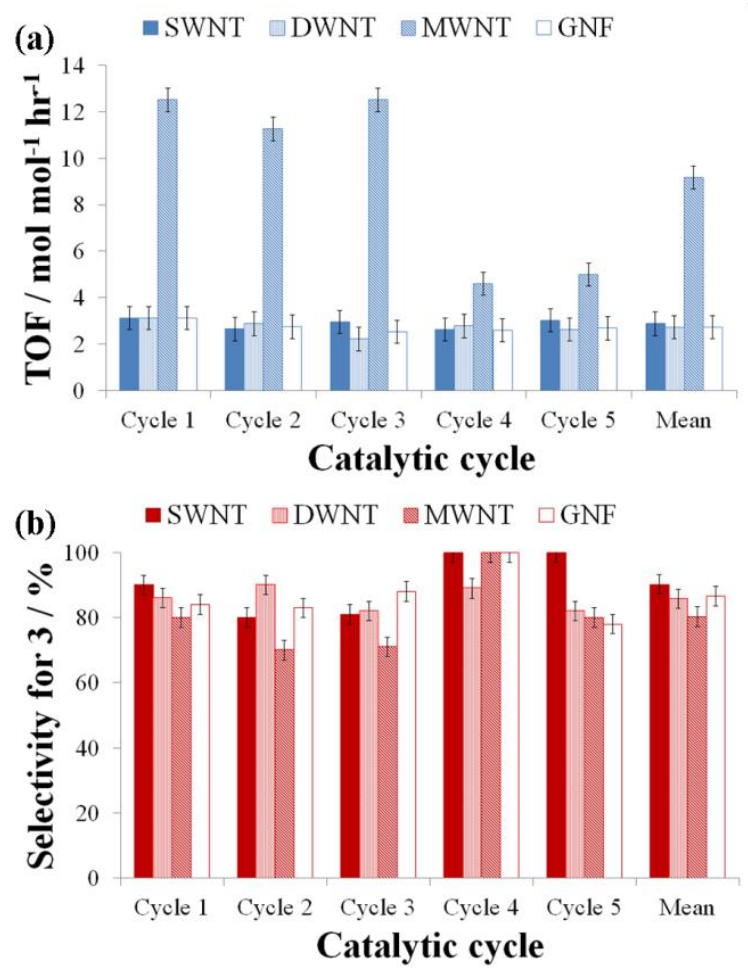

Fig. 3 Comparison of (a) conversion and (b) selectivity for 3 in the Suzuki10 Miyaura cross coupling of 1-iodo-4-nitrobenzene 1c and phenylboronic acid 2 using dodecanethiolate-stabilised PdNP supported on different nanostructured carbon supports. Error bars reflect the standard error associated with the analytical technique.

After the final catalytic cycle in the reaction of $\mathbf{1 c}$ and $\mathbf{2}$, an 15 increase in the mean size of nanoparticles was observed in virtually all cases, the extent of which appeared to be related to the method of preparation, the nature of the stabiliser and the underlying carbon support. For the different nanostructured carbons, the degree of nanoparticle ripening followed the trend SWNT $>>$ 20 DWNT $>$ MWNT GNF (entries 1-2 and 5-6, Table 5). This, coupled with reductions in surface loading, provides compelling evidence that catalyst stability is intimately linked to the curvature of the cylindrical nanostructure, with highly curved surfaces providing the smallest dispersion interactions between 25 nanoparticles and nanotubes and the lowest retardation of the growth and coalescence of catalytic centres and, thus, the lowest catalyst stability. This provides further powerful evidence that the interactions between carbon nanotubes and nanoparticles are dominated by van der Waals forces, which depend on the curvature 30 and polarisability of carbon nanostructures, as opposed to contributions from covalent bonding, related to the pyramidalisation angle and thus reactivity of individual carbon atoms, where the opposite effect would be expected. It is interesting to note, however, that all catalysts based on alkylthiol 35 stabilised nanoparticles showed exceptional catalyst performance despite these dynamic morphological changes across catalytic cycles. Conversely, more drastic changes were observed for the nanoparticles stabilised by other surfactants. For example, total loss of the metal was observed for MWNT/PdNP-4 and ${ }_{40}$ MWNT/PdNP-5 (entries 9-10, Table 5) and therefore these catalysts will not be useable beyond the two measured cycles of catalysis. Significant sintering and aggregation was observed for all other MWNT supported catalysts (entries 7-8 and 11, Table 5) and thus explains the dramatic drop in catalyst performance.

45 A further interesting and unexpected observation from this analysis concerns the relative differences in the morphology of MWNT/PdNP-1 catalysts used in the reactions of the 1-halo-4nitrobenzenes $\mathbf{1}$ and phenylboronic acid 2 (entries 3-5, Table 5, Fig. 4 and $\mathrm{S} 4 \uparrow$ ). For the catalyst involved in the reaction of 150 chloro-4-nitrobenzene 1a, the nanoparticle size and density was largely unaffected, consistent with its inability to participate in the cross-coupling reaction due to the very short $\mathrm{C}-\mathrm{Cl}$ bond. Conversely, the use of the bromo-derivative $\mathbf{1 b}$ results in a 1.8-fold increase in mean nanoparticle size and 13.7-fold decrease in 55 surface density. This effect is also seen, albeit to a significant lesser effect, in the reaction of 1-iodo-4-nitrobenzene $1 c$ and 2 (1.2-fold increase in $\mathrm{d}_{\mathrm{NP}}$, 4.6-fold increase in $\left.\Theta_{\mathrm{NP}}\right)$. Clearly, the nature of the reactive intermediate generated in these two systems (where $\mathrm{X}=$ $\mathrm{Br}$ and $\mathrm{I}$ ) is different. Although the exact active species formed in 60 situ from nanoparticulate catalysts under the conditions of crosscoupling has yet to be irrefutably identified, soluble $[\mathrm{ArPdX}]$ and [ArPdX $]^{-}$species (where Ar $=$aryl, $\mathrm{X}=$ halide), formed by the oxidative addition of $\mathrm{ArX}$ to $\mathrm{Pd}$ surface atoms, have been previously detected in solution and presumed to account for the 

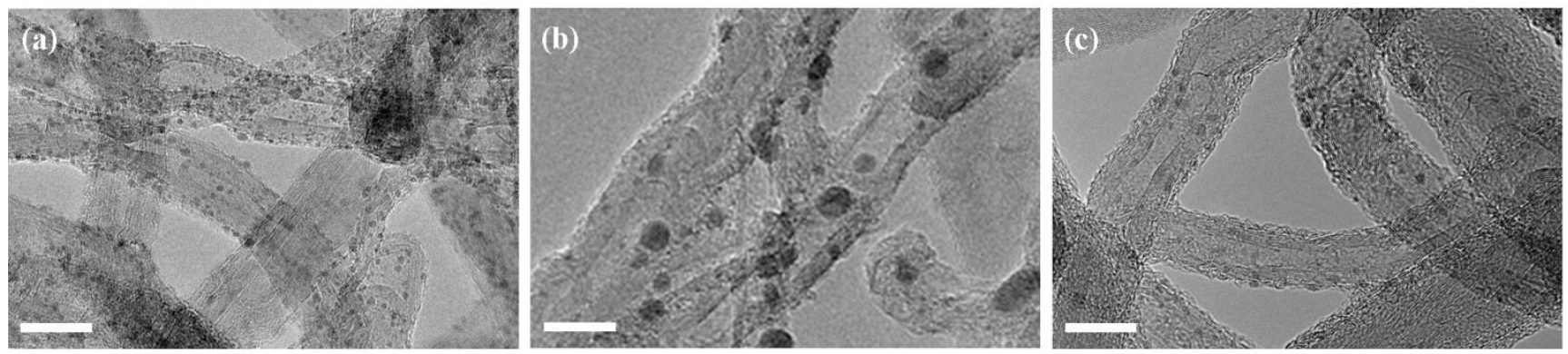

Fig. 4 The effect of the 1-halo-4-nitrobenzene 1 reagent, where (a) $\mathrm{Cl}$, (b) $\mathrm{Br}$ and (c) I, on the size and distribution of catalytic centres subsequent to the Suzuki-Miyuara reaction catalysed by MWNT/PdNP-1. Scale bars are $20 \mathrm{~nm}$.

Table 6 Comparison of the catalytic performance of the CNS/PdNP catalysts in the Suzuki-Miyaura cross-coupling reaction of 1c and $\mathbf{2}$ (Scheme 1).

\begin{tabular}{|c|c|c|c|c|c|}
\hline Entry & Catalyst & Activity & Selectivity & Recyclability & Catalyst performance \\
\hline 2 & DWNT/PdNP-1 & good & good & good & good \\
\hline 4 & MWNT/PdNP-2 & good & moderate & moderate & moderate \\
\hline 5 & MWNT/PdNP-3 & moderate & excellent & poor & poor \\
\hline 6 & MWNT/PdNP-4 & good & good & moderate & moderate \\
\hline 7 & MWNT/PdNP-5 & good & good & moderate & moderate \\
\hline
\end{tabular}

10 of growth (Ostwald ripening) of the palladium nanoparticles observed in our comparative studies must be related to the formation, stability and solubility of the reactive intermediates $\left[\mathrm{ArPdBr}_{2}\right]^{-}$and $\left[\mathrm{ArPdI}_{2}\right]^{-}$. As the ease of Pd insertion into the Ar$\mathrm{X}$ bond increases within the Group, following the order $\mathrm{I}>\mathrm{Br}>\mathrm{Cl}$, 15 the propensity of $\left[\mathrm{ArPdI}_{2}\right]^{-}$to form and subsequently react is significantly greater than the corresponding $\left[\mathrm{ArPdBr}_{2}\right]^{-}$species. Furthermore, the solubility of such intermediates is known to decrease as you go down the group. Thus, the lifetime of the soluble $\left[\mathrm{ArPdI}_{2}\right]^{-}$species in the reaction mixture is significantly 20 decreased relative to the bromine-containing analogue and therefore redeposition onto the parent nanoparticle surface under the known conditions of equilibrium becomes more facile. This is consistent with the observed reduction in reaction time (Table 3). This retards the detrimental growth of nanoparticle catalysts and 25 ensures higher activity over a number of catalytic cycles. This halide-dependency for dynamic Ostwald ripening has important practical applications for catalysis. Whilst it is widely recognised that aryliodides are more reactive in cross-coupling reactions, our study clearly shows that the use of aryliodides (as opposed to 30 arylbromides) is also beneficial for maintaining the catalytically active surface area of PdNP on nanotubes as the detrimental effect of nanoparticle ripening is retarded for $\mathrm{X}=\mathrm{I}$ as compared with $\mathrm{X}$ $=\mathrm{Br}$. The choice of reagent is thus critical for effective and sustained catalyst performance.

35

\section{Conclusions}

We have shown that the catalytic properties of composite nanotube-nanoparticle superstructures critically depend on morphological characteristics expressed at the nanoscale. We have 40 compared a number of complementary approaches for the decoration of carbon nanostructures with palladium nanoparticles and assessed their use as catalytic centres in cross-coupling reactions (Table 6 ).

The deposition of pre-formed dodecanethiolate-stabilised 45 palladium nanoparticles on MWNT yielded the optimum catalytic system, exhibiting high activity and selectivity over multiple catalytic cycles. The catalyst preparation methodology was directly transferable to a range of nanostructured carbon supports, where the effect of nanotube polarisability on uptake of 50 nanoparticles from suspension was clearly evidenced, with larger more polarisable MWNT possessing the highest capacity for palladium nanoparticle adsorption. Systematic microscopic investigation of the composite nanostructures highlighted that nanoparticles increase in size subsequent to catalysis, the extent of 55 which is directly related to the curvature of the underlying carbon support with narrower SWNT unable to retard the growth (Ostwald ripening) of nanoparticles during catalysis owing to the minimised nanoparticle-nanotube interactions. We have discovered important practical implications regarding the choice of reagents for cross60 coupling reactions, as the formation and stability of the reactive intermediates $[\mathrm{ArPdX}]_{2}^{-}$generated in situ critically depend on the nature of the halide, with iodoarenes (i.e. $\mathrm{X}=\mathrm{I}$ ) resulting in reduced ripening of the nanoparticle catalyst and thus prolonged catalytic performance. The findings disclosed in our study are both 65 timely and important as nanoscale catalysts are revolutionising the synthesis of fine chemicals, where the macroscopic catalytic 
properties critically depend on an understanding of fundamental processes at the nanoscale.

\section{Acknowledgements}

ANK is grateful to the ERC for financial support and to the NNNC 5 for access to TEM facilities. BC is grateful for financial support from MIUR (PRIN 2010-2011 - prot. 2010N3T9M4) and "ItalianFrench University" (Vinci 2011), CNRS and the University of Reims. NMR spectroscopic measurements by C. Petermann (University of Reims) are gratefully acknowledged.

\section{${ }_{10}$ Notes and references}

a Dipartimento di Farmacia, Università "G. d'Annunzio", Via dei Vestini, I-66100 Chieti, Italy.

${ }^{b}$ Institut de Chimie Moléculaire de Reims, CNRS UMR 7312, Université de Reims Champagne-Ardenne, UFR Pharmacie, 51 Rue Cognacq-Jay,

15 F-51096 Reims Cedex, France.

${ }^{c}$ School of Chemistry, The University of Nottingham, University Park, Nottingham, NG7 2RD, UK. Fax: +44 115 9513563; Tel: +44 115

9513917; E-mail: graham.rance@nottingham.ac.uk;

andrei.khlobystov@nottingham.ac.uk

$\dagger$ Electronic Supplementary Information (ESI) available: [additional characterisation and catalytic applications of the CNS/PdNP composites]. See DOI: $10.1039 / \mathrm{b} 000000 \mathrm{x} /$

1 C. A. Foss Jr. and D. L. Feldheim, Metal Nanoparticles: Synthesis 25 Characterisation \& Application, Marcel Dekker, New York, 2002.

2 R. Narayanan and M. A. El-Sayed, J. Am. Chem. Soc., 2003, 125, 8340.

3 R. Narayanan and M. A. El-Sayed, J. Phys. Chem. B, 2004, 108, 8572.

4 A. Fihri, M. Bouhrara, B. Nekoueishahraki, J. M. Basset and V.

$30 \quad$ Polshettiwar, Chem. Soc. Rev., 2011, 40, 5181.

5 V. Georgakilas, D. Gournis, V. Tzitzios, L. Pasquato, D. M. Guldi and M. Prato, J. Mater. Chem., 2007, 17, 2679.

6 M. S. Dresselhaus, G. Dresselhaus and P. Avouris, Eds. Carbon Nanotubes: Synthesis, Structure, Properties and Applications, Springer, Berlin, 2001

7 A. Gniewek, J. J. Ziolkowski, A.M. Trzeciak, M. Zawadzki, H. Grabowska and J. Wrzyszcz, J. Catal., 2008, 254, 121.

8 P. Han, X. Wang, X. Qiu, X. Ji, and L. Gao, J. Mol. Catal. A, 2007, 272, 136.

409 A. Monopoli, A. Nacci, V. Caló, F. Ciminale, P. Cotugno, A. Mangone, L. C. Giannossa, P. Azzone and N. Cioffi, Molecules, 2010, 15, 4511.

10 B. M. Coudary, S. Madhi, N. S. Chowdari, M. L. Kantam and B. Sreedhar, J. Am. Chem. Soc., 2002, 124, 14127.

4511 S.-F. Yin, B.-Q. Xu, C.-F. Ng and C.-T. Au, Appl. Catal. B, 2004, 48, 237.

12 S. Akbayrak and S. Özkar, ACS Appl. Mater. Interfaces, 2012, 4, 6302.

13 N. A. Kotov, Nanoparticle assemblies and superstructures, CRC Press, Boca Raton, 2006.

5014 W. M. Tolles, Nanotechnology, 1996, 7, 59.

15 J. Guerra and M. A. Herrero, Nanoscale, 2010, 2, 1390.

16 X. H. Peng, J. Y. Chen, J. A. Misewich and S. S. Wong, Chem. Soc. Rev., 2009, 38, 1076.

17 G. G. Wildgoose, C. E. Banks and R. G. Compton, Small, 2006, 2, 182.

5518 D. Eder, Chem. Rev., 2010, 110, 1348.

19 V. Tzitzios, V. Georgakilas, E. Oikonomou, M. Karakassides and D. Petridis, Carbon, 2006, 44, 848.

20 T. M. Day, P. R. Unwin, N. R. Wilson and J. V. Macpherson, J. Am. Chem. Soc., 2005, 127, 10639.

6021 T. M. Day, P. R. Unwin and J. V. Macpherson, Nano Lett., 2007, 7, 51.

22 X. R. Ye, Y. Lin and C. M. Wai, Chem. Commun., 2003, 642.

23 Z. Sun, Z. Liu, B. Han, S. Miao, Z. Miao and G. An, J. Colloid Interface Sci., 2006, 304, 323.
6524 M. Cano, A. Benito, W. K. Maser and E. P. Urriolabeitia, Carbon, 2011, 49, 652.

25 B. Xue, P. Chen, Q. Hong, J. Lin, K. Lee and K. Tan, J. Mater. Chem., 2001, 11, 2378

26 C.-H. Liu, B.-H. Mao, J. Gao, S. Zhang, X. Gao, Z. Liu, S.-T. Lee, X.70 H. Sun and S.-D. Wang, Carbon, 2012, 50, 3008.

27 F. P. Zamborini, S. M. Gross and R. W. Murry, Langmuir 2001, 17, 481.

28 V. Mazumber and S. Sun, J. Am. Chem. Soc. 2009, 131, 4588.

29 K. A. Flanagan J. A. Sullivan, and H. Müeller-Bunz, Langmuir 2007, $75 \quad 23,12508$.

30 G. A. Rance, D. H. Marsh, S. J. Bourne, T. J. Reade and A. N. Khlobystov, ACS Nano, 2010, 4, 4920.

31 A. Corma, E. Garcia and A. Leyva, J. Molecular Catal. A, 2005, 230, 97.

8032 S. Yang, X. Zhang, H. Mi and X. Ye, J. Power Sources, 2008, 175, 26.

33 N. Karousis, G.-E. Tsotsou, F. Evangelista, P. Rudolf, N. Ragoussis and N. Tagmatarchis, J. Phys. Chem. C, 2008, 112, 13463.

34 H.-B. Pan, C. H. Yen, B. Yoon, M. Sato, and C. M. Wai, Synth Commun., 2006, 36, 3473.

8535 K. Sonogashira, Y. Tohda and N. Hagihara, Tet. Lett., 1975, 4467.

36 R. F. Heck and J. P. Nolley, J. Org. Chem., 1972, 37, 2320.

37 A. Balanta, C. Godard and C. Claver, Chem. Soc. Rev, 2011, 40, 4973.

38 N. Miyaura, K. Yamada and A. Suzuki, Tet. Lett., 1979, 20, 3437.

39 N. Miyaura, T. Yanagi and A. Suzuki, Synth. Commun., 1981, 11, 513.

9040 N. Miyaura and A. Suzuki, Chem. Rev., 1995, 95, 2457.

41 K. Billingsley and S. Buchwald, J. Am. Chem. Soc, 2007, 129, 3359.

42 A. G. Myers, N. J. Tom, M. E. Fraley, S. B. Cohen and D. J. Madar, J. Am. Chem. Soc., 1997, 119, 6072.

43 N. Yasuda, J. Organomet. Chem., 2002, 653, 279.

${ }_{95} 44$ R. W. Friesen, C. Brideau, C. C. Chan. S. Charlesa, D. Deschenes and D. Dube, Bioorg. Med. Chem. Lett., 1998, 8, 2777.

45 M. M. Heravi and E. Hashemi, Tetrahedron, 2012, 68, 9145.

46 J. G. de Vries, Dalton Trans. 2006, 421. 